\title{
Social network predictors of latrine ownership
}

\author{
Holly B. Shakya, Ph.D. ${ }^{a, b}$, Nicholas A. Christakis, MD, Ph.D. ${ }^{b}$, James H. Fowler Ph.D. ${ }^{a c}$ \\ ${ }^{a}$ School of Medicine, University of California, San Diego, 9500 Gilman Dr. La Jolla, CA 92093 \\ hshakya@ucsd.edu: jhfowler@ucsd.edu \\ ${ }^{b}$ Yale Institute for Network Science PO Box 208263 New Haven, CT 06520 nicholas.christakis@yale.edu \\ ${ }^{c}$ Political Science Department, University of California, San Diego SSB 301, 9500 Gilman Dr. La Jolla CA 92093
}

Corresponding author: Holly B. Shakya, hshakya@ucsd.edu, 619-241-3016

(C) 2014. This manuscript version is made available under the Elsevier user license http://www.elsevier.com/open-access/userlicense/1.0/ 


\section{Social network predictors of latrine ownership}

Keywords: India; social networks; social influence; sanitation; south asia; open defecation; social norms; social learning 


\section{$\underline{\text { Abstract }}$}

Poor sanitation, including the lack of clean functioning toilets, is a major factor contributing to morbidity and mortality from infectious diseases in the developing world.

We examine correlates of latrine ownership in rural India with a focus on social network predictors. Participants from 75 villages provided the names of their social contacts as well as their own relevant demographic and household characteristics. Using these measures, we test whether the latrine ownership of an individual's social contacts is a significant predictor of individual latrine ownership. We also investigate whether network centrality significantly predicts latrine ownership, and if so, whether it moderates the relationship between the latrine ownership of the individual and that of her social contacts.

Our results show that, controlling for the standard predictors of latrine ownership such as caste, education, and income, individuals are more likely to own latrines if their social contacts own latrines. Interaction models suggest that this relationship is stronger among those of the same caste, the same education, and those with stronger social ties. We also find that more central individuals are more likely to own latrines, but the correlation in latrine ownership between social contacts is strongest among individuals on the periphery of the network.

Although more data is needed to determine how much the clustering of latrine ownership may be caused by social influence, the results here suggest that interventions designed to promote latrine ownership should consider focusing on those at the periphery of the network. The reason is that they are 1) less likely to own latrines and 2) more likely to exhibit the same behavior as their social contacts, possibly as a result of the spread of latrine adoption from one person to another. 


\section{Background and significance}

\section{The problem of sanitation}

Diarrheal diseases are one of the most common causes of death for children under the age of 5, with almost 50\% of those deaths occurring in India, Nigeria, Pakistan, Democratic Republic of Congo, and China (Black et al., 2010). Poor sanitation, including the lack of clean functioning toilets, is a major factor contributing to these outcomes (Fewtrell et al., 2005; Pruss et al., 2002) and increasing access to sanitation is an important part of the Millennium Development Goals (MDG) (WHO \& UNICEF, 2006). Exposed fecal matter pollutes ground water, drinking water, soil, and food sources (Bonu \& Kim, 2009). Besides its contribution to morbidity and mortality due to diarrheal diseases, exposed fecal matter also spreads diseases like typhoid and parasitic infections caused by worms (Nath, 2003).

Not surprisingly, research shows that increasing access to latrines is a significant way to decrease diarrheal morbidity for children under 5 in India (Kumar \& Vollmer, 2011), although, importantly, the effect of a child's individual household latrine ownership is relatively insignificant compared to the effect of increasing communitywide latrine ownership. For instance an analysis by Spears found that open defecation accounts for much of the childhood stunting in India (Spears, 2013). In some ways, latrine ownership is a classic network good, creating positive externalities for others beyond the actual owner and user. However, for an individual latrine user the results of those positive externalities (i.e. lower rates of diarrhea and other fecal-transmitted infectious diseases, lower rates of stunting) may be hard to observe and/or hard to attribute to the proportion of her village that uses latrines, and therefore the spread of latrines may rely more on the change of normative influences than on the observed health benefits of adoption.

Although India has made considerable progress in increasing the number of latrines around the country, close to $74 \%$ of the population still defecates in the open (Bonu \& Kim, 2009). Rural access to latrines in the south Indian state of Karnataka, from which the data for this study was collected, is consistent with that of the rest of the country. While approximately $75 \%$ of urban households have access to latrines, only $17 \%$ of those in rural areas have access (Baligar, 2006), although government sponsored latrine building campaigns in the last decade have increased that number to approximately 30\% through some areas of the state (Meenakshisundaram, 2008).

Socio-economic predictors of household latrine ownership in India are not unexpected. Besides living in an urban area, those with the most education and highest incomes are the most likely to have latrines (Bonu \& Kim, 2009; Dickinson \& Pattanayak, 2012; Veerashekharappa \& Bhide, 2009). These factors affect both the resources to build a latrine as well as the exposure to, and understanding of, reasons why having a latrine would be beneficial. Caste may also serve as a proxy for many of these factors (Bonu \& Kim, 2009). Those of the scheduled castes and scheduled tribe groups have historically been marginalized both socially and economically, and, consistent with these characteristics, they are the groups least likely to own latrines (Bonu \& Kim, 2009; 
Veerashekharappa \& Bhide, 2009). It has been suggested however, that these groups are also geographically stymied with respect to latrine ownership given housing situations with little access to the kind of drainage necessary for a properly functioning latrine (Bonu \& Kim, 2009). In some areas, it has also been found that Hindus are less likely to own latrines, as they believe that latrines situated close to their residences are polluting and find the latrines themselves "disgusting" (Meenakshisundaram, 2008).

More nuanced studies have found that, even controlling for more obvious demographic factors, social norms and social expectations may drive latrine building decisions (Pattanayak et al., 2009). For instance, results from a qualitative study in Benin highlighted the fact that latrine-building decisions were primarily rooted in motivations such as prestige, and had little to do with concerns regarding health (Jenkins \& Curtis, 2005). Indian villagers have reported preferring open defecation because it allows people the chance to chat together, or because it is a time honored custom in their community (Dickinson \& Pattanayak, 2012). Results from a study assessing the effects of Community Led Total Sanitation (CLTS) demonstrated that galvanizing entire communities was a necessary aspect of a latrine adoption campaign, and that the adoption decisions of the entire community were one of the strongest influences in a household's decision to build a latrine (Dickinson \& Pattanayak, 2012). The most successful latrine building campaigns have been those initiated by CLTS that successfully shift the norms of the community towards intolerance of open defecation, and community willingness to invest in building latrines (Dyalchand et al., 2008). While the Indian government has increased its efforts to provide latrine coverage for the nation, modeling its programs after CLTS, it has been unsuccessful at achieving positive results for those living in the most poverty, those belonging to marginalized castes, and those living in rural areas (Bonu \& Kim, 2009). Government subsidies for building latrines have been largely unsuccessful as deeper social forces, including caste-based social divides, seem to affect the adoption of latrine usage within communities (Chambers, 2009; Lamba \& Spears, 2013). Pattanayak and colleagues (2009) have found, in fact, that for those above the poverty line, social shaming is a more economical and efficacious strategy for promoting latrine adoption than the use of subsidies, although monetary support may be necessary for those below the poverty line.

\section{Perspectives on social effects}

Research on social norms has highlighted the difference between "descriptive norms", which are prevalent behaviors within a community, and "injunctive norms" or norms that are enforced within a community through sanctions - either positive sanctions for behaving within normative expectations, or negative sanctions for normative violations (Cialdini et al., 1991; Lapinski \& Rimal, 2005). As new norms begin to become entrenched in a community, there is often a tipping point, or a point at which a high enough proportion of the population has adopted the new process that from there on it begins to spread more rapidly, such that it may seem like an instantaneous change (Burke \& Young, 2009; Sunstein, 1996).

In some developing world communities, the practice of open defecation may be simply a descriptive norm, and persist because it is commonly practiced. In other areas, however the practice may be an injunctive norm, and those who attempt to transgress 
may be ridiculed or criticized. For instance, researchers in Kenya discovered that normative taboos around defecation include the belief that the feces of a father-in-law and daughter-in-law should not mix together, and therefore open defecation is a means by which this restriction is protected (Bwire, 2010). In order to discourage people from practicing open defecation, programs such as CLTS are attempting to foster injunctive norms to create an atmosphere of shame for those who defect from appropriate sanitation practices (Dyalchand et al., 2008). With this strategy, community members who defecate in the open receive negative sanctions, such as ridicule and public shaming. Villages are encouraged to be "open-defecation free," a process that requires all of the members of a community to change their behavior as well as monitor the behavior of others.

Social contagion, a term which refers to the mechanism by which social behaviors can diffuse through a population, can differ according to the type of norm held in place. Social learning is more likely to take place in the context of descriptive norms, when individuals observe others engaging in a behavior which seems beneficial, such as having a latrine for their household (Kohler et al., 2001; Montgomery \& Casterline, 1996). The fact that others have taken the risk to engage in the behavior and seem to be accruing benefits from it makes it easier for the individual to adopt the behavior themselves. On the other hand, social influence processes are those that occur when behaviors are encouraged or constrained due to injunctive norms. The CLTS programs are designed with the idea that social influence dynamics can be cultivated in order to abolish the practice of open defecation within villages. Individuals are afraid to defect because to do so would risk the disapprobation of those in their social networks.

Social network analysis can be used to elucidate some of the nuances of these processes. Research on networks has suggested that many behavioral processes are in fact the function of network dynamics. Broadly speaking, two main network mechanisms can impact the behavioral decision of any individual: connection and contagion.

Contagion occurs when information or behaviors spread through a network from individual to individual. Recent research on network contagion has suggested that a wide variety of health-related and social behaviors and outcomes may spread from person to person through social networks in both observational and experimental settings (Christakis \& Fowler, 2007; Christakis \& Fowler, 2012; Fowler \& Christakis, 2010; Rosenquist et al., 2010). These dynamics have been observed up to three degrees of separation, and they may also transcend homogeneous peer-to-peer network relations(Shakya et al., 2012).

Network studies that look at connection offer additional insight by demonstrating how the structural position of an individual might impact that individual's behavior. Centrality measures, for instance, indicate which individuals are most connected within a network, and are positively correlated with their ability to influence others, and their tendency to be influenced (Christakis \& Fowler, 2010b; Christley et al., 2005; Gayen \& Raeside, 2010b; Rothenberg et al., 1995)

Intervention strategies are increasingly focused on targeting the most central individuals with the idea that they will be able to positively influence others to adopt an innovation (Fujimoto et al., 2009; Valente, 2005a). For example, Banerjee and colleagues (2013) studied the network diffusion of the decision to participate in a microfinance program among villagers in rural India. They found that the overall participation in microfinance programs was significantly higher when the introduction of 
information occurred among more centrally positioned individuals (Banerjee et al., 2013). On the other hand, the most central individuals are also at a higher risk if the behavior or outcome being transmitted through the network is negative such as substance abuse, or an infectious disease(Christakis \& Fowler, 2010b).

\section{This study}

Few studies have quantitatively characterized the predictors of latrine ownership in developing country settings, and while the results of qualitative studies suggest that the success of latrine building campaigns seem to hinge on socially driven factors, even fewer studies have examined the relationship between social network characteristics and latrine ownership. A working paper by Dickinson and Pattanayak (2012) used the results of a randomized control trial of the CLTS program strategy to assess to what degree social factors drive latrine adoption. They found that, after accounting for a wide range of village and household characteristics, a household is more likely to adopt a latrine if their neighbors do. Similarly researchers in Benin found that neighborhood levels of latrine adoption were significantly associated with an increased probability that households in those neighborhoods would build a latrine (Jenkins \& Cairncross, 2010).

While these studies are suggestive of network effects, none used social network data in which direct connections between individuals could be mapped and analyzed (Smith \& Christakis, 2008). With social network data, connections between individuals are directly reported rather than inferred, allowing the calculation of direct rather than indirect effects between individuals and their social contacts: in other words we can statistically model the possibility of contagion. Of equal importance, having complete network data offers the opportunity to analyze the potential role of connection. Network centrality has been found to facilitate the spread of a new innovation in some contexts, while constraining that spread in others (Gayen \& Raeside, 2010a; Valente, 2005b). The analysis of network centrality offers researchers a new tool in the quest to understand the relationship between social dynamics and health behavior as well as the efficacy of health behavior interventions.

Here, we use network data collected from 75 villages in rural Karnataka to test whether having social contacts with latrines is associated with the probability that an individual will have a latrine. Going beyond these measures, we also test whether a person's place in the overall network is significantly associated with their probability of latrine ownership. While being central or peripheral to the network may impact an individual's chance of owning a latrine, potentially through increased exposure to latrine building norms or interventions, it may also act to moderate the relationship between the latrine ownership of an individual and that of their social contacts, illustrating an interaction between connection and contagion effects. While the observational nature of the data makes it impossible to be certain about causality, our results can nevertheless inform future research on this topic.

\section{Methods}

\section{Data}

We use sociocentric network data collected by Abhijit Banerjee and colleagues from 75 villages in rural Karnataka, in Southern India (Banerjee et al., 2013; Jackson et 
al., 2012). Sociocentric studies focus on a small population and attempt to ascertain all of the social relationships within a set of interconnected individuals(Marin \& Wellman, 2011). This is in contrast to egocentric network studies that focus on a larger population and attempt to ascertain all of the social relationships of a set of randomly chosen individuals that are usually not connected to one another. Whereas egocentric data may help to improve the representativeness of a sample for a large population, sociocentric data allows measurement of larger network structures (like communities) and individual level network measures based on them.

In Karnataka, data were collected in 2006-2007 as part of a study to understand the network diffusion of micro-finance. A complete census was taken by interviewing one person within each household within the village, regarding household characteristics such as latrine ownership, and roof construction. Individual surveys were then used to collect demographic and network data from over one half of eligible households (those with women between the ages of 18 and 57), which were randomly chosen using stratification by religion and geographic sub-location (Banerjee et al., 2013). Respondents included eligible women within each household and their spouses. The total number of individuals interviewed was 16,984. After removing observations with missing data, our analysis included data on 16,579 individuals coming from 6811 households or approximately $46 \%$ of all households per village. Of these, 6543 were household heads, 5919 were spouses of household heads, and 4117 were other individuals in the household. We excluded any network data representing ties in which a surveyed individual nominated a non-surveyed individual. We defined our population as being eligible women and their spouses, and links to others outside this population, like any sociocentric network study, were unavoidably excluded. An additional feature was the sampling on such women; but work by Banerjee and colleagues (2013) suggests that such sampling, especially at such a high sampling fraction, does not meaningfully bias network statistics

Participants reported their age, gender, religion (Hindu or Muslim), and mother tongue (Kannada, Tamil, Telugu, or Hindi). Participants were also asked to identify to which caste they belong (scheduled caste, scheduled tribe, obc (other backward caste), or general). Education was measured using 16 levels ranging from none to higher degree. Household quality variables included roof type (6 categories), number of rooms in the home, number of beds in the home, and household electricity (private electricity, government electricity, or no electricity). Consistent with prior work in traditional agrarian societies in which data regarding actual income is unreliable, we used these household quality measures as a proxy for income (Morris et al., 2000). An additional income measure was the type of ration card held by each individual. Ration cards are used in India to guarantee government subsidies for food depending upon income, which we categorized as BPL (below poverty level), ABL (above poverty level), and not holding a card.

Our outcome variable was a binary measure of household latrine ownership.

\section{Network measures}

A "name generator" is the survey instrument used in social network data collection to elicit the important ties of individuals (Marin, 2004). In this study, respondents (here termed egos) were asked to name up to 8 individuals (here termed 
alters) for each name generator, and there were 12 name generators administered. These included asking respondents who they: 1) borrow money from, 2) give advice to, 3) help with a decision, 4) borrow kerosene or rice from, 5) lend kerosene or rice to, 6) lend money to, 7) obtain medical advice from, 8) engage socially with, 9) are related to, 10) go to temple with, 11) invite to one's home, 12) visit in another's home.

Because previous research has shown that networks derived from multiple name generators can more successfully measure network characteristics related to network composition (Marin \& Hampton, 2007), we collated data from all 12 name generators to create one comprehensive network. Each network tie indicated that one individual had named the other (or vice versa) in at least one name generator. We then calculated tie strength by summing the total number of name generators in which one of them named the other. This gives us a way to quantify multiplexity, or the degree to which the relationship between two people encompasses different types of exchange or interaction(Feld, 1981). The resulting data set yielded an undirected weighted network in which the weight on each tie ranged from 1 to 12 .

Using the igraph library in $\mathrm{R}$, we calculated three individual level network centrality measures for each individual in each village. Degree (Proctor \& Loomis, $1951)$ is simply the total number of unique social contacts that nominate or are nominated by the respondent. Closeness centrality (Sabidussi, 1966) is the inverse of the average social distance between a respondent and all other people in the network, where the social distance between friends is 1 , between friends of friends is 2 , and so on. To make its scale comparable to other variables, we transform it by multiplying it by 100 . Betweenness centrality (Freeman, 1977), is the number of shortest paths in the network that pass through a respondent divided by the total number of shortest paths. This measure identifies the extent to which an individual in the network is critical for passing anything from one individual to another. The higher this number, the greater the effect would be on the total average distance for the network if this person were removed (Wasserman \& Faust, 1994). Because of the skewed distribution of betweenness, we transform it by adding 1 and taking the log.

Homophily is the tendency for individuals to form social ties with others that are similar to them (McPherson et al., 2001). In this case, we wanted to test whether the correlation between ego and alter's latrine ownership varied according to homophily on significant characteristics. We created binary measures for each dyad: one to assess whether the respondent and her social contact were of the same caste, and one to assess whether they had the same level of education.

\section{Statistical Analyses}

Our final dataset consisted of one observation for each ego-alter dyad, including pertinent covariates for both individuals. This dyadic model provided us the most precision when including covariate information for both the ego and the alter (as opposed to a model with one observation per ego and averaged measures for the alters). We first conducted bivariate analyses to test associations with ego latrine ownership using simple logistic regression and $\chi^{2}$ tests. Variables that were at least marginally significant in the bivariate analyses $(p<0.10)$ were included in a multivariate model, for parsimony. We then interacted significant $(p<0.05)$ individual level covariates and the two homophily measures with the main predictor (alter's latrine ownership). To account for village level 
factors that may contribute to possible latrine ownership, such as geography or exposure to government latrine building campaigns, we included village level fixed effects in all of our multivariate models.

To account for autocorrelation that may arise from multiple observations of the same household, we used generalized estimating equation (GEE) procedures that cluster on household and assume an independent working correlation structure for the clusters. The GEE regression models in the tables provide parameter estimates in the form of beta coefficients, whereas the results reported in the text are in the form of odds ratios. We include many controls in these models, but the key coefficient is alter's latrine ownership (see Table 2).

\section{Results}

Summary statistics for the sample population are presented in Table 1 . The mean age was 39 , with approximately $54 \%$ of participants female. Roughly $31 \%$ of the sample was scheduled caste $(25 \%)$ and scheduled tribe $(6 \%)$, groups who have historically been the most disadvantaged and marginalized. The great majority of the participants were Hindu. Thirty-eight percent of the participants had no education, with the mean number of years of education being approximately 5 (SD 4.64). The mean number of participants per village was 221 (SD 70). There were a total of 117,340 dyadic observations used in the analyses with a mean degree centrality score of 9.1 (SD 4.72). Some $30 \%$ of homes had a latrine, which is higher than the $17 \%$ average in rural India as a whole. The 2011 census shows the overall proportion of households with a latrine in Karnataka to be $51.2 \%$ although this varies widely from $11.2 \%$ in the district of Yadgir to $94.8 \%$ in the district of Bangalore (India, 2011). The majority of the homes had private electricity $(66 \%)$, with $29 \%$ using government electricity and $6 \%$ having no electricity at all. Table 1 shows the proportion of individuals in each demographic group having a latrine. Latrine ownership is higher among those with higher caste status, more education, private electricity, and more technologically advanced roof construction.

We present network characteristics in Table 1. Each village was a discrete network and as such network characteristics were separately calculated for each village. Villages were strongly connected units, with almost all participants included in single village wide components. The only exceptions to this were 8 pairs of isolate dyads, who were connected only to each other and were later removed from the dataset as same household pairs.

Figure 1 shows the network of a randomly chosen village from the dataset. Lower caste individuals (scheduled caste and scheduled tribe) are depicted by diamonds while higher caste individuals (obc and general) are depicted by circles. Latrine owners are orange and non-latrine owners are purple. The size of an individual node is proportionate to the proportion of that individuals social contacts that own a latrine. Discrete clusters of high and low caste individuals are readily apparent in the figure, as are clusters of latrine owners vs. non-latrine owners. It is also apparent from this figure that latrine owners are more likely to be higher caste.

While the results of the bivariate analysis (see Table 2) suggest that almost all of the important predictors are statistically significant, when added together in a multivariate analysis (see Table 3) the effects of language, religion, and all of the roof type categories (save RCC), diminish to non-significance (see Figure 2). Consistent with prior research, 
those of the highest castes have the highest probability of owning a latrine versus those of the scheduled caste (OR 1.83, CI 1.41-2.38 for OBC and OR 2.90, CI 1.98-4.25 for General Caste). For each increase in education category, the odds of owning a latrine are 1.07 (CI 1.05-1.08) times that of the lowest education level. Those considered above the poverty level have an odds of latrine ownership 2.24 times higher (CI 1.81-2.78) than those considered below the poverty level. Household level predictors of latrine ownership are having private electricity versus having none (OR 3.28, CI 1.65-6.54)), RCC rooftype versus thatch (OR 5.26, CI 1.73-15.9), more rooms in the house (OR 1.40, CI 1.27-1.55), and more beds (OR 1.24, CI 1.10-1.38).

In the bivariate analysis, there is a strong correlation between ego's latrine ownership with alter's latrine ownership (see 3). The odds of ego owning a latrine if alter does are 2.44 (CI 2.25-2.64)) times the odds of ego owning a latrine if alter does not. While this effect decreases in the multivariate model (OR 1.27 CI 1.16-1.39), it remains significant even when accounting for caste, education, religion, language, poverty level, household level income indicators, and village level fixed effects. Figure 1 shows the relationship between latrine owners and non-latrine owners within the network of one village within the dataset. Latrine owners are more likely to have friends who own latrines, evidenced by their larger node sizes.

In order to test the validity of our results we ran several falsification and robustness tests. First, when we include non-significant variables in the model, the observed relationship between ego and alter latrine ownership does not diminish (See SA Table SA1). Second, to guard against the possibility that our results occurred by chance, we ran 200 permutations in which we randomly assigned ego/alter connections within each village while maintaining the degree distributions for both egos and alters. (See SA Table SA2). We then reran our main analysis for each of these permutations. The results show that the latrine correlations between the permuted pairs are insignificant, suggesting that our original results are robust. Third, the significant relationship between ego's and alter's latrine ownership also persists even when including another level of analysis in which we tested the full multivariate model plus all of alter's covariates (see SA Table SA3). Fourth, to understand whether our significant results were the outcome of our model specifications, we ran the model again using linear regression. The results did not change (see SA Table SA4). Fifth and finally, to tease out possible differential effects in the relationship between ego's and alter's latrine ownership depending upon which name generator was used, we ran 12 separate models for each network by name generator (see SA Table SA5). We found that for all but one name generator, the relationship between ego's and alter's latrine ownership is significant, although the effect size varies slightly from OR 1.19 (CI 1.07-1.31) for with from whom do you take advice to 1.42 (CI 1.241.63) for with whom are you related.

To see whether there are factors that influence the strength of the association between ego and alter latrine ownership, we included interactions in the model between alter's latrine ownership and other variables. Interactions with ego's caste and education level were not significant, suggesting that these variables do not influence the effect alter may have on ego (See SA table SA6). However, the interaction tests for both of the homophily measures were significant. If ego and alter were the same caste, or had the same level of education then the association between ego's and alter's latrine ownership was slightly but significantly greater (Table 2). Our measure of tie strength also 
significantly increases the association between ego and alter's latrine ownership (see Table 2). In other words, the more multiplex the ties, the greater the probability that ego owns a latrine if alter does. Although this is not a causal test, it is consistent with other work that suggests social contacts with strong ties are more likely to influence one another than those with weak ties (Bond et al., 2012).

An interesting comparison test is to study whether there is an association between ego and alter for other household assets besides latrines. The dataset generally lacks these kinds of variables, but we were able to duplicate our analyses using household roof type. We find that the correlation between alter's roof type and ego's roof type is significant, but in contrast to our latrine results, there were no differential effects related to multiplexity, same caste category, or same education category for roof type (see SA Table SA7). This means that egos and alters with stronger social ties and more social similarity are no more likely to own the same kind of roof, and it suggests that social processes may be less important to the choice of roof type than they are for latrines.

In our final set of models we tested measures of network centrality: namely degree, betweenness, and closeness. Because these measures tend to be highly correlated with each other (See SA Table SA8) we ordered our models so as minimize the risk of collinearity. We first ran bivariate regressions for each network measure. We then ran separate multivariate regressions (multivariate meaning the inclusion of covariate controls and fixed effects) for each network measure. To ensure then that our centrality results were not being driven by degree, we then ran separate models for betweenness and closeness centrality controlling for degree. Our final models were then the interactions.

Degree showed a strong correlation with ego latrine ownership in the bivariate model, but, in the multivariate model, this association disappeared. In contrast, betweenness centrality and closeness centrality remained significant even when including numerous controls including degree centrality (see Table 3 and SA Table 9 for models run including non-significant variables). Each standard deviation increase in centrality is associated with increased odds of owning a latrine of 1.14 (95\% CI 1.08-1.20) for betweenness, and 1.20 (95\%CI 1.17-1.23) for closeness.

We also found significant interaction effects for both centrality measures with alter's latrine ownership. While those with the highest centrality measures were most likely to own a latrine, higher centrality measures also diminished the association with alter's latrine ownership. To better understand this dynamic, we stratified the data and repeated the analyses for those who were at the highest and lowest quartiles of both measures. We find that alter having a latrine increases the odds of individual latrine ownership by 1.46 (95\% CI 1.25-1.71) for those with the lowest betweenness centrality scores versus an odds of 1.13 (95\% CI 1.04-1.22) for those with the highest betweeness centrality. This is even more pronounced for closeness centrality. For those with a low closeness centrality score alter having a latrine increases the odds of ego having one by 1.65 (95\% CI 1.41-1.93) versus an odds of 0.97 (95\% CI 0.88-1.07) for those of high closeness centrality (See Figure 4). If a causal process underlies these associations, it suggests that people at the center of the network are less likely to be influenced by their social contacts than people at the periphery. Figure 1 shows that latrine owners with a smaller proportion of their friends who own latrines tend to be more central (the small 
orange nodes in the center) while those with a larger proportion of their friends who own latrines tend to be more peripheral (the larger orange nodes on the outskirts).

\section{$\frac{\text { Discussion }}{W e}$}

We have identified a novel association between latrine ownership and social network characteristics in a rural, developing-world setting. Using sociocentric data from 75 villages in rural India, we tested whether the latrine ownership of a person's social contacts significantly predicted their own latrine ownership. We also explored whether the social network characteristics of an individual increased the probability of owning a latrine.

Controlling for caste, education, income, and village-level fixed effects, we find that a person is significantly more likely to own a latrine if their social contacts also own latrines. We also find that homophily may help to explain some of this association: similarity between ego and alter in caste and education increase the likelihood that they exhibit the same outcome (they both own a latrine or neither owns a latrine). Furthermore, we also find that multiplexity plays a role in this association. Specifically, people who have social ties in multiple dimensions are more likely to exhibit similarity in latrine ownership. We did not, on the other hand, find this relationship with multiplexity for roof type..

The strong relationship between the latrine ownership of ego and that of alter raises important questions regarding possible causal mechanisms. While the crosssectional nature of the data precludes firm conclusions regarding causality, we can explore hypotheses that might offer important clues. It is possible to differentiate between two plausible mechanisms by which peer influence effects might take place (Fujimoto et al., 2009; Marsden \& Friedkin, 1993).

With cohesion, we would expect evidence that direct peer-to-peer contact is the source of knowledge and norms transmission. In other words, people who are directly connected are directly influencing each other through one-on-one interaction (Burt, 1987). On the other hand, structural equivalency occurs when two people adopt the same behaviors not because they themselves are necessarily directly connected, but because they are connected to the same people. In this scenario, it is assumed that ego and alter adopt the same behavior because they are vying for position within their greater social network. The greater the similarity between ego and alter with respect to their social ties, and their relative standing amongst those ties, the greater the competition. So ego is likely to adopt a behavior exhibited by alter if ego feels that the adoption of this behavior will give alter an edge in his/her relationship with others in their shared network.

While our dyadic models provide evidence of cohesion, they do not rule out the possibility of structural equivalency. We therefore ran an additional test for structural equivalency (Table SA 10), with the results indicating that the relationship between ego and alter latrine ownership is most likely driven by direct relationships rather than those shared with other people (i.e., there was no evidence of structural equivalency as an explanation).

Adding network measures to the models yielded several further results. First, we found that people with stronger ties exhibit greater similarity in latrine ownership. This is consistent with other work that suggests close friends influence each other more than weak friends (Brown \& Reingen, 1987) but it is also possible that people who are more 
similar (reflected by their ownership of latrines) are more likely to become close. Second, we found that network-level indicators of connectivity (as indicated by betweenness and closeness centrality) were strong predictors of latrine ownership, even net of numerous controls for socioeconomic status, and net of the number of social contacts (degree). It is not just the popularity of an individual that matters; it is also the individual's structural position within the network as whole. Third, while those who are most central are most likely to own a latrine, they are also potentially less likely to be influenced by their social contacts than those at the periphery. This raises an interesting dilemma for efforts to promote adoption: should we target people who are more influential at the center of the network or people who are potentially more influenceable at the periphery (Christakis \& Fowler, 2010a)?

There are several possible explanations for these results. Government-sponsored latrine building campaigns were implemented in Karnataka during the 2000's, though not all areas were uniformly covered (Meenakshisundaram, 2008). However, the rate of household latrine ownership in our overall sample is consistent with that achieved after the government's sanitation campaign, suggesting that government-sponsored toilet building interventions took place in these villages. The high rate of latrine ownership among the most central individuals may thus be explained by the tendency for those most central in a community to receive the most exposure to outside interventions. This is both because their social position naturally offers them greater exposure to innovations spreading in the network, and also because many interventions attempt to target people who may be the most central believing them to be socially influential based on the observable traits of such individuals (Borgatti et al., 2009; Fujimoto et al., 2009; Valente, 2010).

People at the center of the network who have not yet adopted may thus be the least influenceable, which is consistent with previous research showing that central actors and those in denser networks can often be the most constrained by prevalent norms (Gayen \& Raeside, 2010a; Kohler et al., 2001; Rosenquist et al., 2010). If latrine adoption has not become normative amongst the majority of the community (which is probable given that only $30 \%$ of households in this sample have latrines), then those nonlatrine owning central individuals may be the most difficult to persuade.

Thus, contrary to common assumptions about the role of centrality in the adoption of innovations, our results suggest that latrine-building interventions targeting those more peripheral to the network may be surprisingly efficacious. Not only are those more peripheral less likely to own a latrine, but they are also more likely to have friends without latrines and more susceptible to external interventions. Most importantly, intervention efforts aimed at the more peripheral community members will potentially have larger multiplier effects, as the correlations between ego and alter latrine ownership are much higher in these groups.

Qualitative studies assessing community-led sanitation programs have pointed to the importance of social norms in the success of programs aiming to increase latrine ownership and usage around the world. For the most part, educational health campaigns and government subsidies have failed due to the inability of such approaches to address the social factors involved in latrine adoption, and have focused on convincing and educating individuals rather than groups. While we cannot definitively conclude that our results reflect the role of normative dynamics, they are suggestive of the possibility. 
While government-sponsored latrine building programs were unsuccessful at creating normative change, a minority of the population adopted latrines, some of them perhaps due to a social learning dynamic, having observed the benefits accrued to a friend or relation who did so. Overall societal norms, however, did not seem to shift to create the sort of social influence required to tip the majority into adopting latrine building.

Finally, while our results are consistent with previous research showing that latrine ownership is differentially distributed by caste, they also suggest that the potential social effect we observed may be stronger for people who are the same caste. This means that the inherent level of social divisiveness that exists within Indian villages may impede the spread of latrine adoption relative to other parts of the world.

\section{Limitations of this study:}

The data used for this analysis was observational and cross-sectional so it is important to be cautious about inferring causation. However, because associations remain significant even when we have accounted for the major predictors of latrine ownership such as caste and education, future research using longitudinal data and experimental designs to investigate possible causality seems warranted.

Due to the static nature of the data, it is also difficult to control for homophily or the possibility that egos and alters are connected due to similarity in the kinds of characteristics that predict latrine ownership. This has long been recognized as a challenge in understanding the nature of social effects and diverse analytic approaches to the problem have been proposed (e.g., Christakis and Fowler, 2007; Christakis and Fowler, 2012). Manski, in a seminal paper on this topic, differentiates between endogenous effects (social influence), exogenous effects (common influences) and correlated effects (homophily) (Manski, 1993). We find some evidence that social similarity may moderate the strength of association in latrine ownership between ego and alter, but it is not possible to determine whether this is because similar people choose to socialize with one another, or if it is because similar people are more liable to be influenced by one another (Centola, 2010). Ideally we would like to control for similarities at an initial point in time and then assess the correlations in latrine ownership over the course of a social relationship.

A further limitation is the lack of village-level measures, meaning we cannot identify which environmental factors may be driving some of the results. Higher rates of latrine ownership were predicted in Benin among larger villages and those closer to urban centers (Jenkins \& Cairncross, 2010), and in Orissa among those who had been exposed to higher quality government latrine campaigns (Dickinson \& Pattanayak, 2012). However, by controlling for village-level fixed effects, we have been able to statistically account for those unmeasured predictors, so they should not affect the estimates of individual and household level effects that we present here. Finally, this data does not measure actual latrine use. While latrine ownership is certainly an important step towards actually using a latrine, it is not a guarantee. Work with CLTS highlighted the fact that it takes more than actually owning a latrine to ensure that people are really using it (Dyalchand et al., 2008).

\section{Conclusion}


To our knowledge, this is the first study to quantitatively characterize the social network characteristics associated with latrine ownership. While an enormous amount of time and energy is being devoted to promoting latrine building and use in South Asia, little quantitative evidence exists to inform programs and policies. Our results suggest that social dynamics may be an integral aspect of latrine adoption and that these dynamics possibly require a more complex approach than simply identifying the most central individuals and appealing to them. Qualitative studies have suggested that for latrine building to succeed it must become normative within a community rather than a matter of individual preference. While further studies are certainly warranted to make any definitive recommendations along these lines, our results are consistent with those conclusions.

\section{References}

Baligar, S.V.P. (2006). Human Development Report Karnataka 2005

. In http://planningcommission.nic.in/plans/stateplan/sdr pdf/shdr kar05.pdf

(Ed.): Planning and Statistics Department Government of Karnataka.

Banerjee, A., Chandrasekhar, A.G., Duflo, E., \& Jackson, M.O. (2013). The Diffusion of Microfinance. science, 341.

Black, R.E., Cousens, S., Johnson, H.L., Lawn, J.E., Rudan, I., Bassani, D.G., et al. (2010). Global, regional, and national causes of child mortality in 2008: a systematic analysis. The Lancet, 375, 1969-1987.

Bond, R., Fariss, C., Jones, F., Settle, A., Marlow, C., \& Fowler, J.H. (2012). A massive scale experiment in social influence and political mobilization.?

Bonu, S., \& Kim, H. (2009). Sanitation in India: Progress, Differentials, Correlates, and Challenges. In A.D. Bank (Ed.), South Asia Occasional Paper Series No. 2.

Mandaluyong City: Asian Development Bank

Borgatti, S.P., Mehra, A., Brass, D.J., \& Labianca, G. (2009). Network analysis in the social sciences. science, 323, 892-895 \%@ 0036-8075. 
Brown, J.J., \& Reingen, P.H. (1987). Social ties and word-of-mouth referral behavior. Journal of Consumer Research, 350-362 \%@ 0093-5301.

Burke, M.A., \& Young, H.P. (2009). Social Norms. In B.J. Bisin Alberto, Jackson Matthew (Ed.), Handbook of Social Economics. Amsterdam: North Holland.

Burt, R.S. (1987). Social Contagion and Innovation: Cohesion versus Structural Equivalence. American Journal of Sociology, 92, 1287-1335.

Bwire, B. (2010). Breaking shit taboos: CLTS in Kenya. In T.I.I.f.E.a. Development (Ed.), Participatory Learning and Action pp. 91-96 \%@ 1357-1938X).

Centola, D. (2010). The spread of behavior in an online social network experiment. science, 329, 1194-1197 \%@0036-8075.

Chambers, R. (2009). Going to Scale with Community-Led Total Sanitation: Reflections on Experience, Issues and Ways Forward. IDS Practice Papers, 1, 01-50

Christakis, N.A., \& Fowler, J.H. (2007). The spread of obesity in a large social network over 32 years. New England journal of medicine, 357, 370-379

Christakis, N.A., \& Fowler, J.H. (2010a). Contagion in prescribing behavior among networks of doctors. Marketing Science, 30, 213-216.

Christakis, N.A., \& Fowler, J.H. (2010b). Social network sensors for early detection of contagious outbreaks. PloS one, 5, e12948 \%@ 11932-16203.

Christakis, N.A., \& Fowler, J.H. (2012). Social Contagion Theory: Examining Dynamic Social Networks and Human Behavior. Statistics in Medicine, 32, 566-577.

Christley, R.M., Pinchbeck, G.L., Bowers, R.G., Clancy, D., French, N.P., Bennett, R., et al. (2005). Infection in social networks: using network analysis to identify high-risk individuals. American journal of epidemiology, 162, 1024-1031.

Cialdini, R.B., Kallgren, C.A., \& Reno, R.R. (1991). A focus theory of normative conduct: A theoretical refinement and reevaluation of the role of norms in human behavior. Advances in experimental social psychology, 24, 1-243.

Dickinson, K., \& Pattanayak, S. (2012). Open Sky Latrines: Do social effects influence technology adoption in the case of a (very) impure public good? . Working Paper, Duke University.

Dyalchand, A., Khale, M., Vasudevan, S., \& Kale, N. (2008). What Communication and Institutional Arrangements Influence Sanitation Related Social Norms in Rural India? In M. L, \& M. S (Eds.), Shit Matters: The Potential of CommunityLed Total Sanitation London: Practical Action.

Feld, S.L. (1981). The Focused Organization of Social Ties. American Journal of Sociology, 86, 1015-1035.

Fewtrell, L., Kaufmann, R.B., Kay, D., Enanoria, W., Haller, L., \& Colford Jr, J.M. (2005). Water, sanitation, and hygiene interventions to reduce diarrhoea in less developed countries: a systematic review and meta-analysis. The Lancet infectious diseases, 5, 42-52.

Fowler, J.H., \& Christakis, N.A. (2010). Cooperative behavior cascades in human social networks. Proceedings of the National Academy of Sciences, 107, 53345338.

Freeman, L.C. (1977). A set of measures of centrality based on betweenness. Sociometry, 35-41. 
Fujimoto, K., Valente, T.W., \& Pentz, M.A. (2009). Network structural influences on the adoption of evidence-based prevention in communities. Journal of Community Psychology, 37, 830-845 \%@ 1520-6629.

Gayen, K., \& Raeside, R. (2010a). Communicative actions, women's degree of social connectedness and child mortality in rural Bangladesh. Child: Care, Health and Development, 36, 827-834 \%@ 1365-2214.

Gayen, K., \& Raeside, R. (2010b). Social networks and contraception practice of women in rural Bangladesh. Social Science \& Medicine, 71, 1584-1592.

India, C. (2011). House, Household Amenities, and Assets.

Jackson, M.O., Rodriguez-Barraquer, T., \& Tan, X. (2012). Social patterns and social quilts: network patterns of favor exchange. American Economic Review, 102, 1857-1897.

Jenkins, M.W., \& Cairncross, S. (2010). Modelling latrine diffusion in Benin: towards a community typology of demand for improved sanitation in developing countries. Journal of water and health, 8, 166-183.

Jenkins, M.W., \& Curtis, V. (2005). Achieving the good life': Why some people want latrines in rural Benin. Social Science \& Medicine, 61, 2446-2459.

Kohler, H.P., Behrman, J.R., \& Watkins, S.C. (2001). The density of social networks and fertility decisions: Evidence from South Nyanza District, Kenya. Demography, 38, 43-58

Kumar, S., \& Vollmer, S. (2011). Does improved sanitation reduce diarrhea in children in

rural India? Health Economics, under revision.

Lamba, S., \& Spears, D. (2013). Caste,'Cleanliness' and Cash: Effects of Caste-Based Political Reservations in Rajasthan on a Sanitation Prize. Journal of Development Studies, 49, 1592-1606.

Lapinski, M.K., \& Rimal, R.N. (2005). An Explication of Social Norms. Communication Theory, 15, 127-147.

Manski, C.F. (1993). Identification of endogenous social effects: The reflection problem. The review of economic studies, 60, 531-542.

Marin, A. (2004). Are respondents more likely to list alters with certain characteristics?Implications for name generator data. Social Networks, 26, 289-307.

Marin, A., \& Hampton, K.N. (2007). Simplifying the Personal Network Name Generator. Field Methods, 19, 163.

Marin, A., \& Wellman, B. (2011). Social network analysis: An introduction. In J. Scott, \& P. Carrington (Eds.), The SAGE Handbook of Social Network Analysis (p. 11). Thousand Oaks, Ca: Sage.

Marsden, P.V., \& Friedkin, N.E. (1993). Network studies of social influence. Sociological Methods \& Research, 22, 127-151.

McPherson, M., Smith-Lovin, L., \& Cook, J.M. (2001). Birds of a feather: Homophily in social networks. Annual Review of Sociology, 415-444

Meenakshisundaram, S. (2008). Feeling the Pulse: A Study of the Total Sanitation Campaign in Five States. New Delhi: WaterAid India. 
Montgomery, M.R., \& Casterline, J.B. (1996). Social learning, social influence, and new models of fertility. Population and Development Review, 22, 151-175 \%@ 0098-7921.

Morris, S.S., Carletto, C., Hoddinott, J., \& Christiaensen, L.J.M. (2000). Validity of rapid estimates of household wealth and income for health surveys in rural Africa. Journal of epidemiology and community health, 54, 381-387

Nath, K.J. (2003). Home hygiene and environmental sanitation: a country situation analysis for India. International Journal of Environmental Health Research, 13, 19-28\%@0960-3123.

Pattanayak, S.K., Yang, J.C., Dickinson, K.L., Poulos, C., Patil, S.R., Mallick, R.K., et al. (2009). Shame or subsidy revisited: social mobilization for sanitation in Orissa, India. Bulletin of the World Health Organization, 87, 580-587

Proctor, C., \& Loomis, C. (1951). Analysis of Sociometric Data. In P. Holland (Ed.), Research Methods in Social Relations. New York: Dryden Press.

Pruss, A., Kay, D., Fewtrell, L., \& Bartram, J. (2002). Estimating the burden of disease from water, sanitation, and hygiene at a global level. Environmental health perspectives, 110, 537-542.

Rosenquist, J., Murabito, J., Fowler, J.H., \& Christakis, N.A. (2010). The spread of alcohol consumption behavior in a large social network. Annals of Internal Medicine, 152, 426.

Rothenberg, R.B., Potterat, J.J., Woodhouse, D.E., Darrow, W.W., Muth, S.Q., \& Klovdahl, A.S. (1995). Choosing a centrality measure: epidemiologic correlates in the Colorado Springs study of social networks. Social Networks, 17, 273-297.

Sabidussi, G. (1966). The centrality index of a graph. Psychometrika, 31, 581-603.

Shakya, H.B., Christakis, N.A., \& Fowler, J.H. (2012). Parental Influence on Substance Use in Adolescent Social Networks. Archives of Pediatrics and Adolescent Medicine, 166.

Smith, K.P., \& Christakis, N.A. (2008). Social networks and health. Annu. Rev. Sociol, 34, 405-429.

Spears, D. (2013). How much international variation in child height can sanitation explain? In W. Bank (Ed.). Washington DC.

Sunstein, C.R. (1996). Social norms and social roles. Columbia Law Review, 96, 903968

Valente, T. (2005a). Network models and methods for studying the diffusion of innovations.

Valente, T. (2010). Social networks and health: Models, methods, and applications: Oxford Univ Press.

Valente, T.W. (2005b). Network models and methods for studying the diffusion of innovations. In P. Carrington, J. Scott, \& S. Wasserman (Eds.), Models and methods in social network analysis pp. 98-116). Cambridge: Cambridge University Press.

Veerashekharappa, \& Bhide, S. (2009). Sanitation Strategies in Karnataka: A Review. Institute for Social and Economic Change.

Wasserman, S., \& Faust, K. (1994). Social Network Analysis: Methods and Applications. Cambridge: Cambridge University Press. 
WHO, \& UNICEF. (2006). Meeting the MGD drinking water and sanitation target: the urban and rural challenge of the decade. In WHO, \& UNICEF (Eds.).

Switzerland.

\section{Figure captions:}

Figure 1: A network depiction of a randomly chosen village from the dataset. Orange nodes are latrine owning and purple nodes are latrine non-owning. Circles are higher caste (OBC and general), diamonds are lower castes (scheduled tribe and scheduled caste). The size of the node increases according to the proportion of the individual's friends that own latrines. Note that: 1) latrine owners are predominantly higher caste (OBC or general) shown by the orange circles 2 ) latrine owners tend to be clustered together as evident by the groupings of orange versus the groupings of purple 3 ) a higher proportion of latrine owners have friends with latrines shown by the high proportion of larger orange nodes 4) those latrine owners who have a smaller proportion of friends with latrines tend to be more central to the network evident by the smaller orange nodes near the center versus the smaller purple nodes near the periphery.

Figure 2: The odds of individual latrine ownership as a function of a 1 standard deviation increase in each social and demographic characteristic, controlling for all other variables in the model including village level fixed effects. For this figure, all categorical predictors (gray bars) were dichotomized. The simplified model used for this figure is shown in SA table 11.

Figure 3: The probability of an individual owning a latrine increases as the proportion of their social contacts owning a latrine increases.

Figure 4: As individual centrality increases, so does the probability of individual latrine ownership (left frame). However, there is an inverse relationship between centrality and the correlation between ego's and alter's latrine ownership (right frame). As individual centrality increases, the correlation between the latrine ownership of an individual and their social contacts decreases. 
Age in years

Education mean years

Number of rooms in house

Number of beds in house

Tie strength ${ }^{\mathbf{S}}$

Total degree centrality

Betweenness centrality

Closeness centrality

Gender (\% female)
Caste

Scheduled caste

Scheduled tribe

OBC

General

Ration card category

Above poverty

Below poverty

Does not own card

Religion

Hindu

Islamic

Language

Kannada

Tamil

Telugu

Hindi

Other

Roof-type

Thatch

Tile

Stone

Sheet

RCC

Other

Household electricity

Private

Govt.

No

Latrine ownership yes

\section{Karnataka India}

$\mathrm{N}=16579$

Mean (SD)

$39.30(12.5)$

$5.02(4.65)$

$2.62(1.54)$

$1.05(1.62)$

$3.12(2.45)$

$9.1(4.72)$

$4.82(1.5)$

$30.7(5.77)$

\section{Proportion of total}

\section{sample}

$55.10 \%$

$25.27 \%$

$6.05 \%$

$56.45 \%$

$12.23 \%$

$17.40 \%$

$66.90 \%$

$15.70 \%$

Proportion with household latrine

$96.11 \%$

$3.79 \%$

$74.92 \%$

$3.98 \%$

$17.33 \%$

$3.59 \%$

$.181 \%$

$30.00 \%$

$12.50 \%$

$12.80 \%$

$37.20 \%$

$44.70 \%$

$55.70 \%$

$22.10 \%$

$38.10 \%$

$29.80 \%$

$46.70 \%$

$31.70 \%$

$23.30 \%$

$22.80 \%$

$48.50 \%$

$43.30 \%$

\section{$1.98 \%$}

$31.38 \%$

$6.69 \%$

$17.06 \%$

$30.55 \%$

$32.57 \%$

$17.96 \%$

$13.74 \%$

$27.89 \%$

$67.47 \%$

$4.11 \%$

$15.21 \%$

$65.53 \% \quad 41.38 \%$

$28.65 \%$

$10.32 \%$

$5.82 \%$

$30.4 \%$

$5.67 \%$ 


\begin{tabular}{|c|c|c|c|c|c|c|c|c|c|c|c|c|c|c|c|}
\hline & \multicolumn{3}{|c|}{ Bivariate analyses } & \multicolumn{3}{|c|}{$\begin{array}{l}\text { Multivariate Model \#1 } \\
\text { main effect }\end{array}$} & \multicolumn{3}{|c|}{$\begin{array}{l}\text { Multivariate Model \#2 } \\
\text { Tie strength interaction }\end{array}$} & \multicolumn{3}{|c|}{$\begin{array}{l}\text { Multivariate Model \# } 3 \\
\text { Same caste interaction }\end{array}$} & \multicolumn{3}{|c|}{$\begin{array}{l}\text { Multivariate Model \#4 } \\
\text { Same educ interaction }\end{array}$} \\
\hline & Beta & SE & $\mathrm{P}$ & Beta & SE & $\mathrm{P}$ & Beta & SE & $\mathrm{P}$ & Beta & SE & $\mathrm{P}$ & Beta & SE & $\mathrm{P}$ \\
\hline Alter's latrine ownership & 0.891 & 0.032 & 0.000 & 0.178 & 0.034 & 0.000 & 0.099 & 0.040 & 0.014 & 0.042 & 0.061 & 0.496 & 0.139 & 0.035 & 0.000 \\
\hline Education in years & 0.126 & 0.005 & 0.000 & 0.062 & 0.006 & $<2 \mathrm{e}-16$ & 0.061 & 0.006 & $<2 \mathrm{e}-16$ & 0.064 & 0.007 & $<2 \mathrm{e}-16$ & 0.060 & 0.007 & $<2 \mathrm{e}-16$ \\
\hline Number of rooms in house & 0.611 & 0.038 & 0.000 & 0.344 & 0.046 & 0.000 & 0.342 & 0.046 & 0.000 & 0.341 & 0.046 & 0.000 & 0.342 & 0.046 & 0.000 \\
\hline Number of beds in house & 0.588 & 0.060 & 0.000 & 0.189 & 0.054 & 0.000 & 0.189 & 0.054 & 0.000 & 0.189 & 0.054 & 0.000 & 0.189 & 0.054 & 0.000 \\
\hline \multicolumn{16}{|l|}{ Ration card } \\
\hline Above poverty line & ref & & & ref & & & & & & & & & & & \\
\hline Below poverty line & -1.512 & 0.078 & 0.000 & -0.749 & 0.098 & 0.000 & -0.744 & 0.098 & 0.000 & -0.745 & 0.098 & 0.000 & -0.745 & 0.098 & 0.000 \\
\hline No ration card & -0.663 & 0.098 & 0.000 & -0.353 & 0.127 & 0.006 & -0.347 & 0.127 & 0.006 & -0.348 & 0.127 & 0.006 & -0.348 & 0.127 & 0.006 \\
\hline \multicolumn{16}{|l|}{ Caste } \\
\hline Scheduled caste & ref & & & ref & & & & & & & & & & & \\
\hline Scheduled tribe & -0.113 & 0.197 & 0.568 & 0.141 & 0.238 & 0.554 & 0.150 & 0.239 & 0.531 & 0.123 & 0.240 & 0.606 & 0.149 & 0.239 & 0.533 \\
\hline OBC & 1.378 & 0.097 & 0.000 & 0.660 & 0.115 & 0.000 & 0.717 & 0.113 & 0.000 & 0.709 & 0.113 & 0.000 & 0.716 & 0.113 & 0.000 \\
\hline General & 1.795 & 0.120 & 0.000 & 1.084 & 0.170 & 0.000 & 1.120 & 0.168 & 0.000 & 1.091 & 0.168 & 0.000 & 1.120 & 0.168 & 0.000 \\
\hline \multicolumn{16}{|l|}{ Household electricity } \\
\hline Private & ref & & & ref & & & & & & & & & & & \\
\hline Govt & -1.842 & 0.098 & 0.000 & -0.956 & 0.113 & $<2 \mathrm{e}-16$ & -0.949 & 0.112 & $<2 \mathrm{e}-16$ & -0.949 & 0.112 & $<2 \mathrm{e}-16$ & -0.950 & 0.112 & $<2 \mathrm{e}-16$ \\
\hline No & -2.391 & 0.334 & 0.000 & -1.438 & 0.298 & 0.000 & -1.434 & 0.297 & 0.000 & -1.436 & 0.297 & 0.000 & -1.433 & 0.297 & 0.000 \\
\hline \multicolumn{16}{|l|}{ Roof-type } \\
\hline Thatch & ref & & & ref & & & & & & & & & & & \\
\hline Tile & 1.131 & 0.444 & 0.011 & -0.284 & 0.497 & 0.568 & -0.267 & 0.497 & 0.591 & -0.269 & 0.497 & 0.588 & -0.267 & 0.497 & 0.591 \\
\hline Stone & 2.020 & 0.443 & 0.000 & 0.563 & 0.503 & 0.263 & 0.568 & 0.502 & 0.258 & 0.565 & 0.503 & 0.261 & 0.568 & 0.502 & 0.258 \\
\hline Sheet & 1.754 & 0.446 & 0.000 & 0.486 & 0.498 & 0.329 & 0.504 & 0.497 & 0.310 & 0.501 & 0.498 & 0.314 & 0.504 & 0.497 & 0.311 \\
\hline $\mathrm{RCC}$ & 3.399 & 0.447 & 0.000 & 1.428 & 0.512 & 0.005 & 1.433 & 0.511 & 0.005 & 1.432 & 0.512 & 0.005 & 1.434 & 0.512 & 0.005 \\
\hline Other & 0.746 & 0.488 & 0.126 & -0.490 & 0.540 & 0.365 & -0.480 & 0.540 & 0.374 & -0.479 & 0.540 & 0.376 & -0.480 & 0.540 & 0.374 \\
\hline \multicolumn{16}{|l|}{ Religion } \\
\hline Hindu & ref & & & & & & & & & & & & & & \\
\hline Islamic & 0.819 & 0.146 & 0.000 & -0.132 & 0.487 & 0.787 & & & & & & & & & \\
\hline \multicolumn{16}{|l|}{ Language } \\
\hline Kannada & ref & & & & & & & & & & & & & & \\
\hline Tamil & -0.403 & 0.170 & 0.017 & -0.213 & 0.212 & 0.315 & & & & & & & & & \\
\hline Telugu & -0.514 & 0.086 & 0.000 & -0.022 & 0.118 & 0.851 & & & & & & & & & \\
\hline Hindi & 0.769 & 0.151 & 0.000 & 0.690 & 0.508 & 0.174 & & & & & & & & & \\
\hline Tie Strength & & & & & & & -0.009 & 0.006 & 0.103 & & & & & & \\
\hline Tie Strength*alter latrine & & & & & & & 0.026 & 0.009 & 0.003 & & & & & & \\
\hline Same caste binary & & & & & & & & & & -0.156 & 0.053 & 0.003 & & & \\
\hline Same caste $^{\star}$ alter latrine & & & & & & & & & & 0.183 & 0.072 & 0.011 & & & \\
\hline Same education binary & & & & & & & & & & & & & -0.086 & 0.040 & 0.029 \\
\hline Same education * alter latrine & & & & & & & & & & & & & 0.219 & 0.051 & 0.000 \\
\hline
\end{tabular}


Table 3: Results of GEE logistic regression analyses using dyadic observations testing the effect of an individual's network characteristics on the probability of

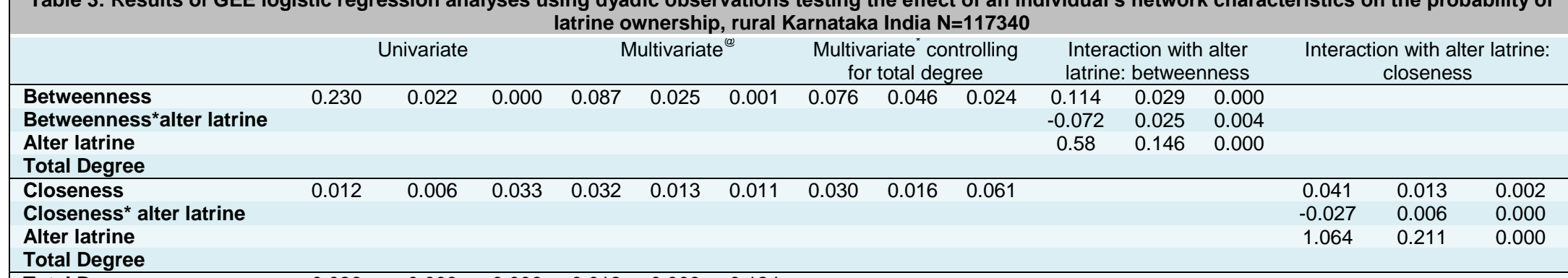

Total Degree

$\begin{array}{lllllll}\text { Total Degree } & 0.026 & 0.009 & 0.006 & 0.012 & 0.008 & 0.124\end{array}$

@Seperate multivariate models were run for each network characteristic with separate demographic controls including respondent caste, education, ration card category, household electricity, household rooftype, number of rooms in house, number of beds in house, alter latrine ownership, and village level fixed effects *Separate multivariate models were run for betweenness and closeness centrality controlling for total degree plus all demographic controls and village level fixed effects. 
Figure1

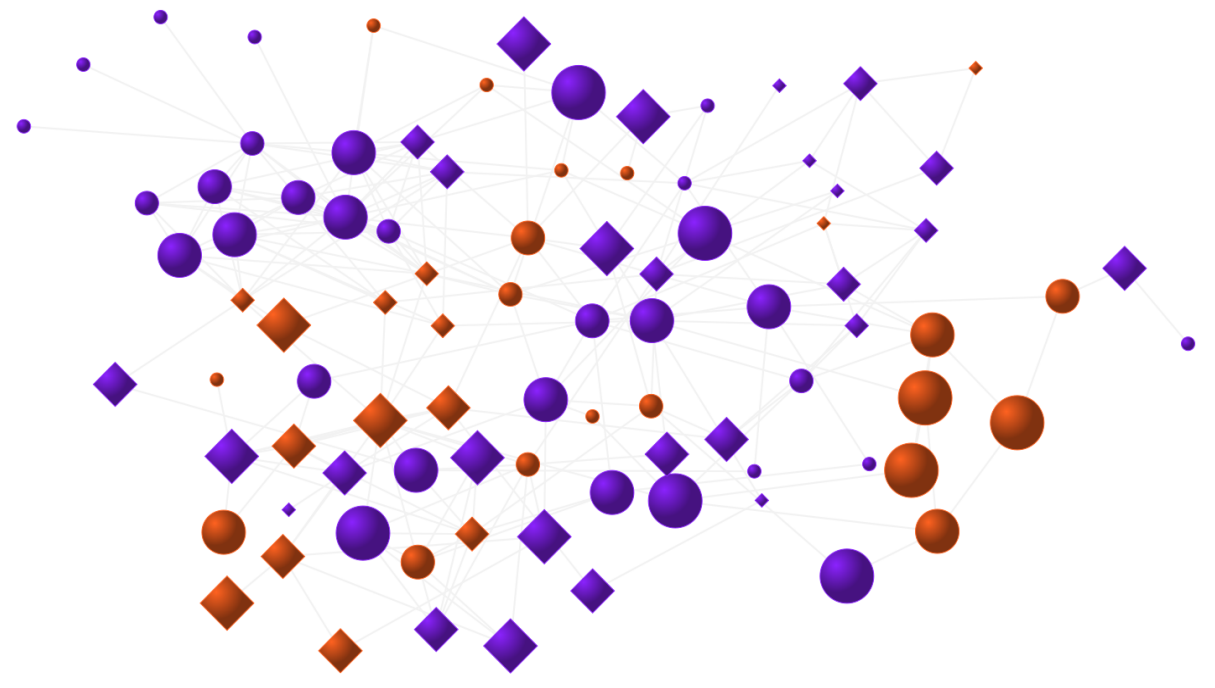


Figure2

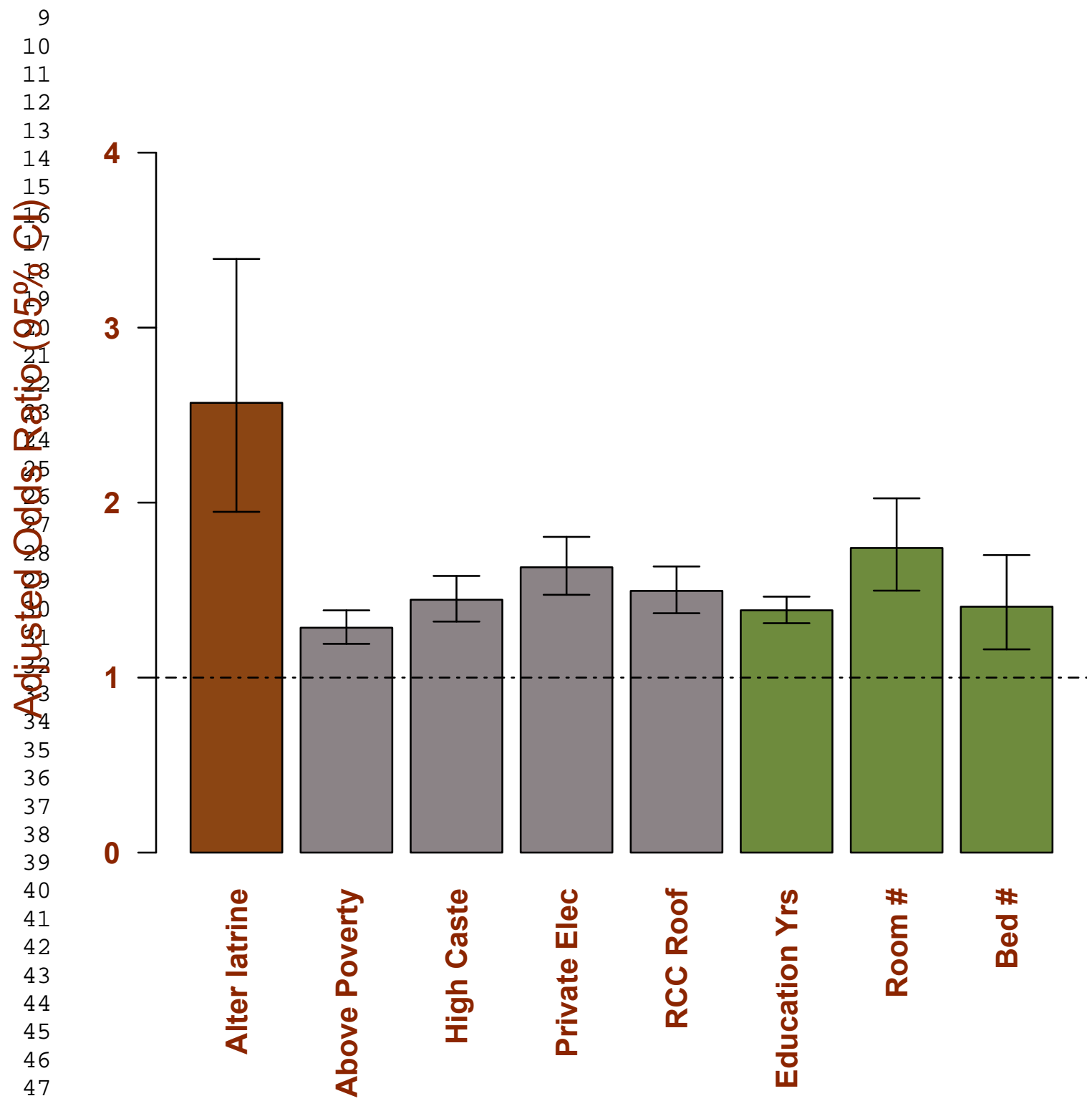

48

49

Social and Demographic Characteristics 
Figure3

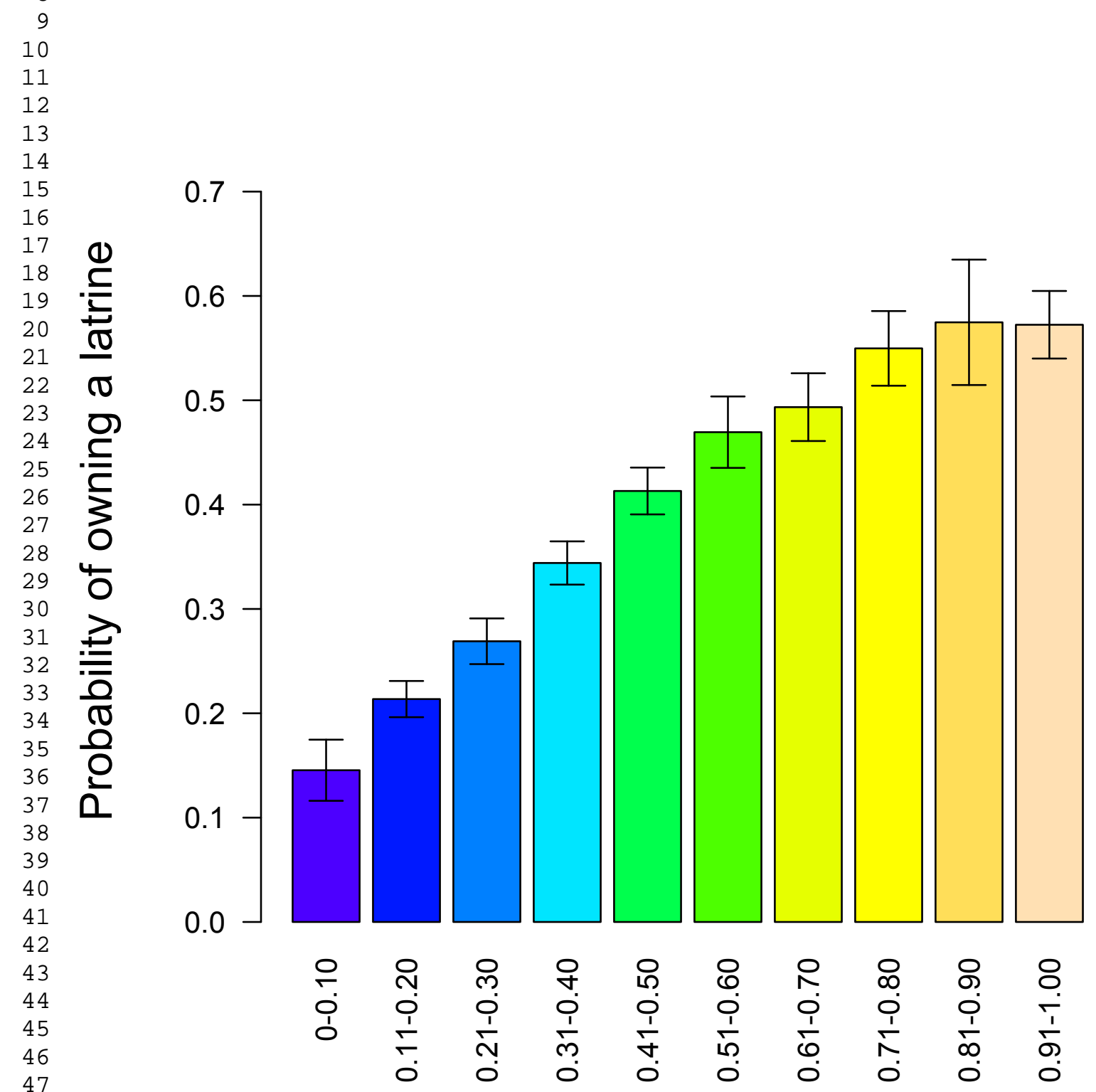

48 49

Proportion of alters with a latrine (per individual ego) 
Figut?

27

28

29

30

.81

部2

इ্छ3

훈

86

8

38

39

$\Phi \odot$

7

82

43

44

45

46

47

48

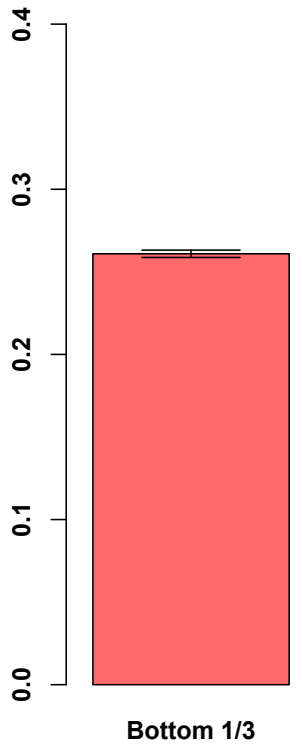

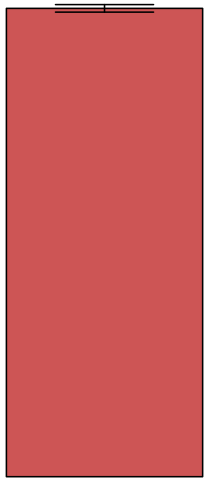

Middle 1/3

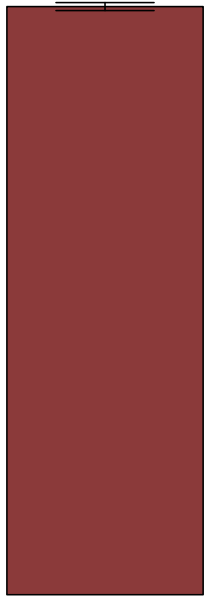

Top 1/3

Ego betweenness centrality

49 


\section{Figut}

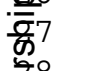

8

है9

$\$ 1$

굴

雪3

बक्षे 4

इ6

\$7

8

\$9 9

Ф०

$\$ 1$

क2

$\frac{0}{4} 3$

$\Phi 4$

\$5

$\$ 6$

$\$ 7$

48
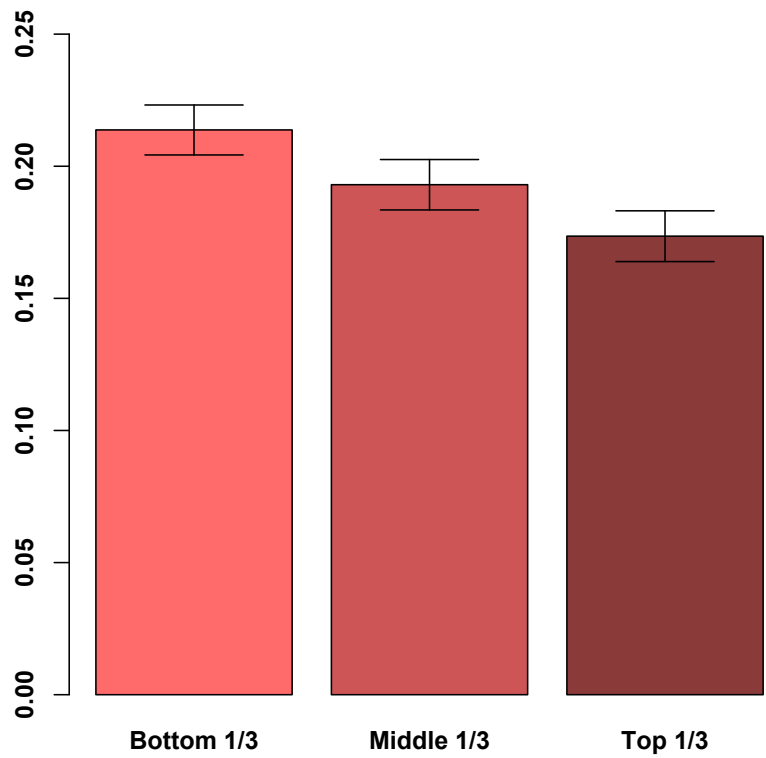

Ego betweenness centrality

49 